\title{
A comparison of tamsulosin and silodosin versus tadalafil in medical expulsive therapy for distal ureteral stone in men: a prospective study
}

\author{
Metin Gur ${ }^{1}$, Suleyman Tumer Caliskan ${ }^{1}$, Muhammet Bahaettin Ulu ${ }^{1}$, Ekrem Akdeniz ${ }^{1}$, \\ and Kemal Ozturk ${ }^{1}$
}

${ }^{1}$ Gazi Hospital

January 13, 2021

\begin{abstract}
Objectives: To evaluate the efficacy of tamsulosin, silodosin, and tadalafil as medical expulsive therapy for distal ureteral stones in men. Methods: Adult males meeting the inclusion criteria were randomized into one of four treatment arms - dexketoprofen (control group), tamsulosin (Group 1), silodosin (Group 2), and tadalafil (Group 3). The stone expulsion rate after four weeks represented the primary endpoint, while the stone expulsion rate at the end of the study and the incidence of adverse events constituted secondary endpoints. Clinical findings were compared between all four drug groups. Results: No statistically significant difference was observed among the groups in terms of age, body mass index, stone futures, expulsion time, pain episodes, or total analgesic use. Expulsion rates in the fourth week were $42.5 \%, 80 \%, 82.5 \%$, and $75 \%$, respectively. The stone expulsion rates in groups 1,2 , and 3 were significantly higher than in the control group $(\mathrm{p}<0.001)$. However, the differences between groups 1, 2, and 3 were not statistically significant. No serious adverse effects were observed during the study period. Conclusion: The study results showed a higher expulsion rate in male patients using tamsulosin, silodosin and tadalafil for distal ureteral stones, but no significant superiority between these. All three are safe, effective, and well-tolerated, causing minimal side-effects.
\end{abstract}

\section{Hosted file}

Main document.pdf available at https://authorea.com/users/389213/articles/503824-acomparison-of-tamsulosin-and-silodosin-versus-tadalafil-in-medical-expulsive-therapyfor-distal-ureteral-stone-in-men-a-prospective-study 

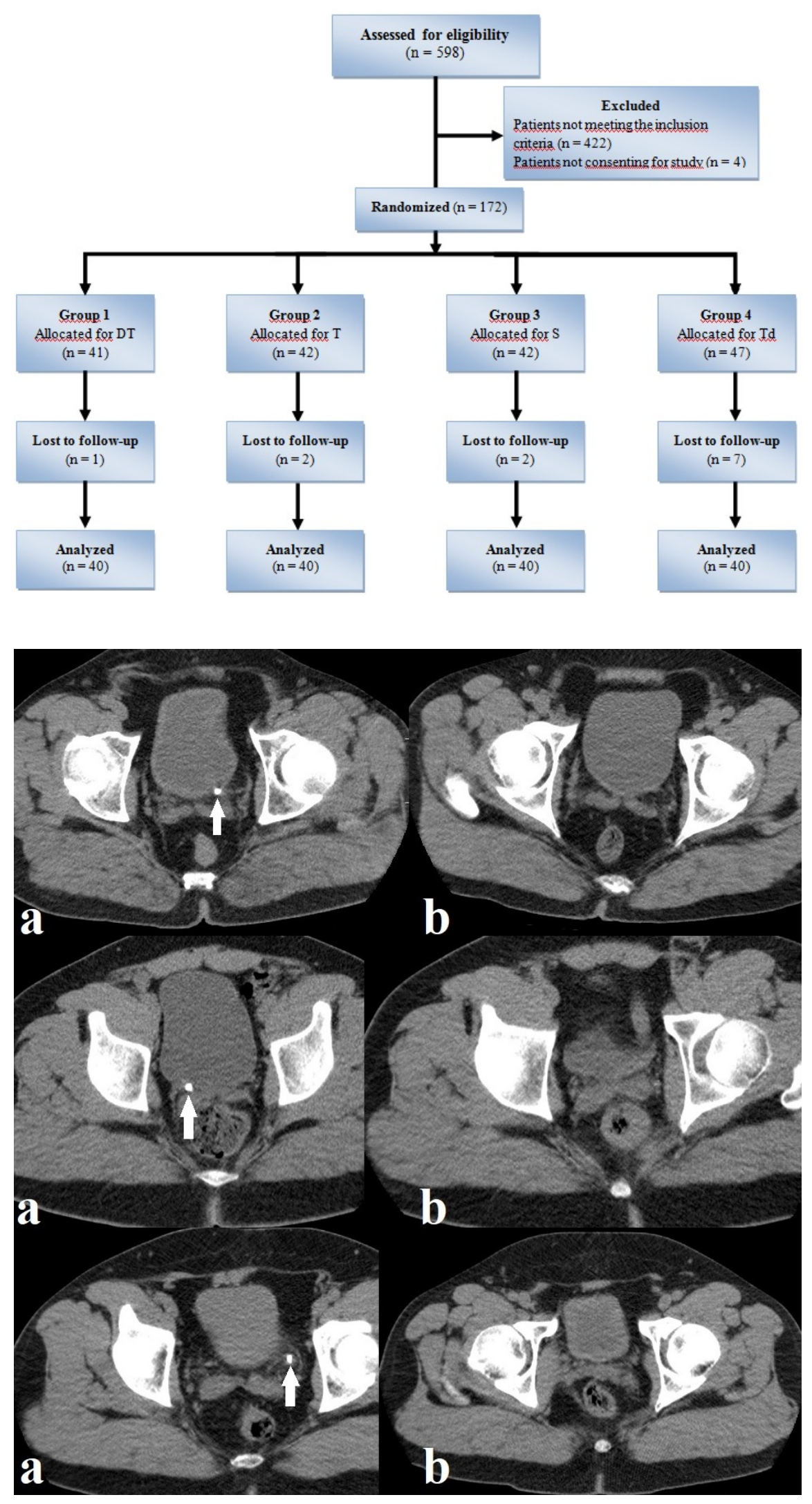